\title{
Direct signals for large extra dimensions in the production of fermion pairs at linear colliders
}

\author{
O. J. P. Éboli, ${ }^{1}$ M. B. Magro, ${ }^{1}$ P. Mathews, ${ }^{2}$ and P. G. Mercadante ${ }^{1}$ \\ ${ }^{1}$ Instituto de Física da USP, C.P. 66.318, São Paulo, SP 05389-970, Brazil \\ ${ }^{2}$ Instituto de Física Teórica, Universidade Estadual Paulista, Rua Pamplona 145, São Paulo, SP 01405-900, Brazil
}

(Received 5 March 2001; published 2 July 2001)

\begin{abstract}
We analyze the potential of the next generation of $e^{+} e^{-}$linear colliders to search for large extra dimensions via the production of fermion pairs in association with Kaluza-Klein gravitons $(G)$, i.e., $e^{+} e^{-} \rightarrow f \bar{f} G$. This process leads to a final state exhibiting a significant amount of missing energy in addition to acoplanar lepton or jet pairs. We study in detail this reaction using the full tree level contributions due to the graviton emission and the standard model backgrounds. After choosing the cuts to enhance the signal, we show that a linear collider with a center-of-mass energy of $500 \mathrm{GeV}$ will be able to probe quantum gravity scales from $0.96(0.86)$ up to 4.1 (3.3) $\mathrm{TeV}$ at a 2 (5) $\sigma$ level, depending on the number of extra dimensions.

DOI: 10.1103/PhysRevD.64.035005

PACS number(s): 12.60.-i, 13.85.Rm, 14.80.-j
\end{abstract}

\section{INTRODUCTION}

Recently there has been great interest in the possibility that the scale of quantum gravity is of the order of the electroweak scale [1] instead of the Planck scale $M_{\mathrm{Pl}}$ $\simeq 10^{19} \mathrm{GeV}$. A simple argument based on Gauss's law in arbitrary dimensions shows that the Planck scale is related to the radius of compactification $(R)$ of the $n$ extra dimensions by

$$
M_{\mathrm{Pl}}^{2} \sim R^{n} M_{S}^{n+2},
$$

where $M_{S}$ is the $(4+n)$-dimensional fundamental Planck scale or the string scale. Thus, the largeness of the fourdimensional Planck scale $M_{\mathrm{Pl}}$ (or smallness of the Newton's constant) can be attributed to the existence of large extra dimensions of volume $R^{n}$. If $M_{S} \sim \mathcal{O}(1 \mathrm{TeV})$, this scenario solves the original gauge hierarchy problem between the weak scale and the fundamental Planck scale, and leads to a rich low energy phenomenology $[1,2]$. The $n=1$ case corresponds to $R \simeq 10^{8} \mathrm{~km}$ for $M_{S}=1 \mathrm{TeV}$, which is ruled out by observations of planetary motions. On the other hand, the $1 / r^{2}$ gravitational force has been shown to hold at separations ranging down to $218 \mu \mathrm{m}$ which corresponds to the bound $M_{S} \gtrsim 3.5 \mathrm{TeV}$ for $n=2$ [3].

Fields propagating in compactified extra dimensions give rise to towers of Kaluza-Klein (KK) states separated in mass by $\mathcal{O}(1 / R)$ [4]. In order to evade the strong constraints on theories with large extra dimensions from electroweak precision measurements, the standard model (SM) fields are assumed to live on a four-dimensional hypersurface, and only gravity propagates in the extra dimensions. This assumption is based on new developments in string theory [5-7]. If gravity becomes strong at the $\mathrm{TeV}$ scale, $\mathrm{KK}$ gravitons should play a role in high-energy particle collisions, either being radiated or as a virtual exchange state ${ }^{1}[2,9-16]$. The KK

\footnotetext{
${ }^{1}$ In the case where brane torsion is considered, which is not mandatory in this model, one can obtain strong bounds for the string scale [8].
}

gravitational modes contain spin-2, spin-1, and spin-0 excitations; however, the spin-1 modes do not couple to the SM particles while the scalar modes couple to the trace of the energy-momentum tensor, therefore not interacting with massless particles.

In this work we study the potentiality of $e^{+} e^{-}$linear colliders (LCs) to probe extra dimensions through the clean and easy-to-reconstruct process

$$
e^{+} e^{-} \rightarrow f \bar{f} \boldsymbol{E}
$$

where the missing energy is due to KK graviton radiation and $f$ can be either a muon or a quark. Since the fermion masses are negligible, only the spin- $2 \mathrm{KK}$ modes are relevant. This process contains not only the $Z^{\star} G$ associated production but also its interference with the $\gamma^{\star} G$ contribution, generalizing the analyses of Ref. [17]. Moreover, we not only apply realistic cuts and include detector resolution effects, but also take into account irreducible backgrounds.

In the framework of the SM, a final state containing lepton pairs plus missing momentum is due to the production of $l \bar{l} \nu \bar{\nu}$ via $Z Z / \gamma$ or $W W$ intermediate states. In the case of jet pairs and missing momentum, there is a large additional contribution due to the production of $q \bar{q}^{\prime} l \nu$ where the extra charged lepton is lost in the beam pipe. The SM also gives rise to two reducible backgrounds via $e^{+} e^{-} \rightarrow f \bar{f}$ or $\gamma \gamma$ $\rightarrow f \bar{f}$ where the momenta of the fermion pair is mismeasured. However, these two backgrounds turn out to be negligible after applying our cuts.

The real KK graviton emission does not interfere with the $\mathrm{SM}$ processes, and consequently, the signal cross section is proportional to $M_{S}^{-n-2}$, with the proportionality constant depending on the number of extra dimensions due the sum over the KK modes. The spin-2 KK graviton radiation gives raise to a very characteristic spectrum of missing energy, and we exploit this feature together with other kinematical distributions to enhance the signal over the SM backgrounds.

This paper is organized as follows. In Sec. II, we describe the techniques used to evaluate the relevant cross sections, while Sec. III contains the main characteristics of the signal 

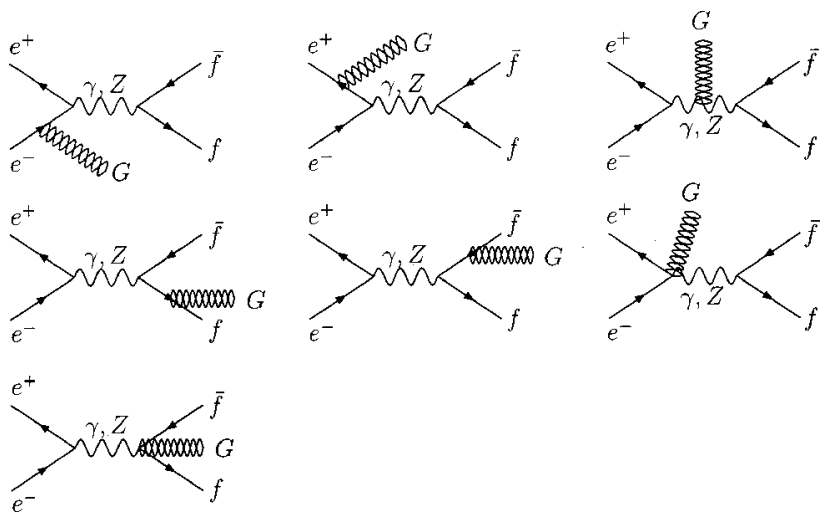

FIG. 1. Feynman diagrams contributing to the KK graviton radiation process $e^{+} e^{-} \rightarrow f \bar{f} G$.

and backgrounds and the cuts chosen to enhance the KK graviton radiation process. In Sec. IV, we present our results and conclusions.

\section{CALCULATIONAL TOOLS}

We are considering the production of fermion pairs accompanied by large energy and momentum imbalances which can be generated by the emission of undetectable KK gravitons. In the case of lepton pairs we analyze only the final state involving muons since the inclusion of taus will present just a small gain in the limits due to the loss in the detection efficiency. On the other hand, the process involving electrons is described by an other class of Feynman diagrams besides the ones computed here, thus deserving an analysis by itself.

The signal and backgrounds were simulated with full tree level matrix elements and, for the hadronic case, we took into account all quark contributions with the exception of the top quark. The spin-2 KK graviton emission is described by 14 Feynman diagrams, see Fig. 1, where the KK graviton is attached to each of the SM fields and vertices appearing in the SM process $e^{+} e^{-} \rightarrow f \bar{f}$. Our notations and Feynman rules for the KK graviton interactions are the ones in Ref. [12], and we have calculated the signal cross section analytically using FORM [18]. On the other hand, the background matrix elements for $e^{+} e^{-} \rightarrow f \bar{f} \nu \bar{\nu}$ and $q \bar{q}^{\prime} l \nu$ were generated using the package MADGRAPH [19]. For the background process $e^{+} e^{-} \rightarrow q \bar{q}^{\prime} l \nu$ special care is required in the phase space integration due to a collinear divergence that appears when the final lepton is an electron or a positron. In this case we employed the prescription for the phase space given in Ref. [20].

In our analyses, the kinematical region of the $f \bar{f}$ pair is such that it is possible that they originate from the on-shell production of $Z$ 's, therefore, we must regulate the $Z$ exchange diagrams in such a way that we do not spoil the gauge invariance of the scattering amplitude. We chose to regulate the $Z$ propagator by introducing finite width effects. In principle, this presents an apparent problem as the matrix elements are not gauge invariant when we simply add an imaginary part in the the $Z$ propagator. Though a formal prescription should be able to take care of this problem, we resorted to an approximation that is extremely reliable in such cases $[16,21]$. The prescription is to multiply an overall factor of the form

$$
\frac{\left(s-M_{Z}^{2}\right)^{2}}{\left(s-M_{Z}^{2}\right)^{2}+\left(M_{Z} \Gamma\right)^{2}} \frac{\left(m_{f \bar{f}}^{2}-M_{Z}^{2}\right)^{2}}{\left(m_{f \bar{f}}^{2}-M_{Z}^{2}\right)^{2}+\left(M_{Z} \Gamma\right)^{2}}
$$

to the summed squared matrix element and not to include the finite width at the matrix element level. Here, $s$ stands for the total center-of-mass energy while $m_{f \bar{f}}$ is the invariant mass of the final state fermion pair. This factor introduces a BreitWigner resonance for the $Z$ boson and for regions far away from the $\mathrm{Z}$ resonance it is essentially of the order of unity.

In order to check our signal matrix element, we verified that it is gauge invariant. Writing the total $Z(\gamma)$ amplitude as $\mathcal{M}^{\alpha \beta} \epsilon_{\alpha \beta}^{*}(k)$, where $\epsilon_{\alpha \beta}(k)$ is the polarization vector of a KK graviton of momentum $k$, we explicitly showed that $\mathcal{M}^{\alpha \beta} k_{\alpha}$ independently vanishes for both $Z$ and $\gamma$ exchange diagrams. Furthermore, we used the gauge invariance to simplify the expression for the cross section, though it remained huge.

We included in our analyses the energy losses due to the emission of photons off the initial state, which we treated in the structure function formalism [22]. The differential cross section is then given by

$$
d \sigma=\int d x_{1} d x_{2} f_{e \mid e}\left(x_{1}, \sqrt{s}\right) f_{e \mid e}\left(x_{2}, \sqrt{s}\right) d \hat{\sigma}\left(\hat{s}=x_{1} x_{2} s\right)
$$

where $\hat{\sigma}(s)$ is the cross section in the absence of initial state radiation, and

$$
f_{e \mid e}(x, \sqrt{s})=\beta\left[(1-x)^{\beta-1}\left(1+\frac{3}{4} \beta\right)-\frac{\beta}{2}(1+x)\right],
$$

with $\beta=\left(\alpha_{\mathrm{em}} / \pi\right)\left[\log \left(s / m_{e}^{2}\right)-1\right]$, is the leading-log resummed effective $e^{ \pm}$distribution function. Note that we did not include beamstrahlung, which is expected to further smear out the peak in the $e^{+} e^{-}$luminosity at $\hat{s}=s$, since it depends on details of accelerator design.

For strongly interacting final states, we simulated the resolution of the hadronic calorimeter by smearing the energies (but not directions) of all final state partons with a Gaussian error given by

$$
\left.\frac{\delta E}{E}\right|_{\mathrm{had}}=\frac{0.30}{\sqrt{E}} \oplus 0.01,
$$

where $E$ is given in $\mathrm{GeV}$.

\section{SIGNAL AND BACKGROUND PROPERTIES}

We started our analyses imposing the following set of acceptance cuts: (C1) We required that the events present a missing transverse momentum $\not p_{T}>10 \mathrm{GeV}$; (C2) the muons or jets should have a transverse momentum $p_{T}$ $>5 \mathrm{GeV}$; (C3) we also required that the muons/jets be ob- 
TABLE I. Total signal cross section in $\mathrm{fb}$ for the muonic channel $\left(\sigma_{\mu \mu E}^{\text {signal }}\right)$ and hadronic channel $\left(\sigma_{j j E}^{\text {signal }}\right)$ for different number of extra dimensions, using $\sqrt{s}=500 \mathrm{GeV}$ and $M_{S}=1 \mathrm{TeV}$ after applying the acceptance cuts $(\mathrm{C} 1)-(\mathrm{C} 4)$, as explained in the text. For comparison, the total cross sections for the SM backgrounds are $\sigma_{\mu \mu \mathrm{k}}^{\text {back }}$ $=73.6 \mathrm{fb}$ and $\sigma_{j j t}^{\text {back }}=3562 \mathrm{fb}$.

\begin{tabular}{|c|c|c|c|c|c|c|}
\hline$n$ & 2 & 3 & 4 & 5 & 6 & 7 \\
\hline$\sigma^{\text {signal }}(\mathrm{fb})$ & 55.1 & 17.2 & 6.08 & 2.27 & 0.888 & 0.357 \\
\hline$\sigma_{j j E}^{\substack{\text { signal } \\
j \text {. }}}(\mathrm{fb})$ & 723 & 203 & 64.4 & 21.9 & 7.82 & 2.86 \\
\hline
\end{tabular}

served in the region $|\cos \theta|<0.98$, where $\theta$ is the muon or jet polar angle; (C4) the jets (muons) are required to be separated by $\Delta R>0.4$, where $(\Delta R)^{2}=(\Delta \eta)^{2}+(\Delta \phi)^{2}$, with $\eta$ being the pseudo-rapidity and $\phi$ the azimuthal angle.

In Table I, we display the total signal cross section after these initial cuts for a center-of-mass energy of $500 \mathrm{GeV}$, assuming $M_{S}=1 \mathrm{TeV}$ and the number of extra dimensions $n=2-7$. We can see from this table that the signal cross section drops quickly as we increase $n$, as expected. After the acceptance cuts $(\mathrm{C} 1)-(\mathrm{C} 4)$, we found that the cross section of the SM background $e^{+} e^{-} \rightarrow f \bar{f} \nu \bar{\nu}$ is $\sigma_{\mu \mu E}^{\text {back }}=73.6 \mathrm{fb}$ in the muonic case and $\sigma_{j j k}=285.8 \mathrm{fb}$ in the hadronic case. For the hadronic final state, the presence of a collinear singularity leads to a large cross section for the process $e^{+} e^{-}$ $\rightarrow q \bar{q}^{\prime} e \bar{\nu}$ where the $e^{ \pm}$is lost in the beam pipe. After cuts (C1) $-(\mathrm{C} 4)$ and requiring $\left|\cos \theta_{e^{+}\left(e^{-}\right)}\right|>0.98$ in order to miss the $e^{ \pm}$into the beam pipe, the cross section for this reaction is $\sigma_{j j \in}^{\text {coll }}=3276 \mathrm{fb}$, making this the dominant background in the hadronic case. Consequently, we needed to introduce further cuts to take care of this background, rendering the analyses of the hadronic case quite different from the muonic one. We finally checked that when a muon or tau is lost into the beam pipe instead of an $e^{ \pm}$, this reaction does not give a significant contribution to the total background after our acceptance cuts due to the absence of collinear divergences.

The dramatic drop of the signal cross section for large values of $n$ compels us to refine our analyses by studying kinematical distributions in order to determine further cuts to enhance the signal. In Fig. 2 we display the missing invariant mass $\left(M_{\text {miss }}\right)$ distributions of the SM backgrounds and KK graviton emission signal for the muonic case after imposing the acceptance cuts and taking $M_{S}=1 \mathrm{TeV}$ and $n=3$. Although they are experimentally indistinguishable, we show the SM background distributions classified by the final neutrino flavor in order to see in detail their behaviors. The electron and tau neutrino flavors present a peak near the $Z$ mass, since they originate from the $Z Z$ contribution to this process, while the distribution due to the muon neutrino is broader, because of the contribution of two $W$ production to this final state. Moreover, the electron neutrino background mimics the signal distribution due to $W$ fusion process. Of course, we added up all backgrounds to obtain the final results. Notice that the dips in the distributions at high $M_{\text {miss }}$ values are due to the acceptance cuts. Clearly, $M_{\text {miss }}$ will be an important variable to reduce the SM background, and

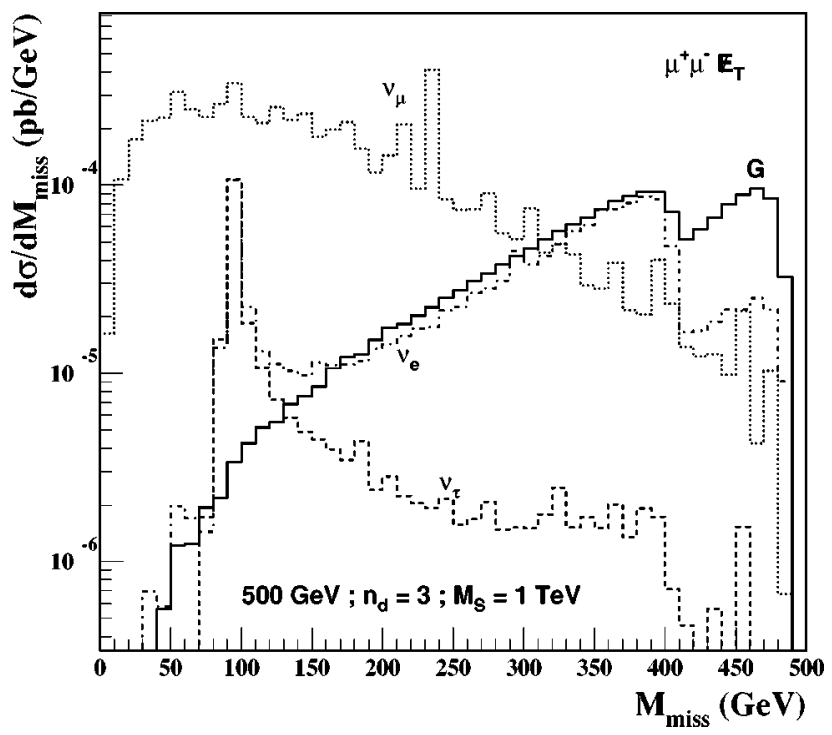

FIG. 2. Missing invariant mass $\left(M_{\text {miss }}\right)$ spectrum originated from the KK graviton radiation (solid line) and the SM contributions to the muonic channel divided in the neutrino flavors: $\nu_{e}$ (dotdashed), $\quad \nu_{\mu}$ (dotted), and $\nu_{\tau}$ (dashed). We assumed $\sqrt{s}$ $=500 \mathrm{GeV}, M_{S}=1 \mathrm{TeV}, n=3$, and applied the acceptance cuts (C1) $-(\mathrm{C} 4)$ described in the text.

consequently we required (C5) $M_{\text {miss }}>320 \mathrm{GeV}$.

Due to the KK graviton emission the final state jets and muons are not expected to be back-to-back. Figure 3 contains the distribution of the cosine of the angle between the final state muons $\left(\cos \theta_{\mu \mu}\right)$ after imposing cuts $(\mathrm{C} 1)-(\mathrm{C} 5)$ for $M_{S}=1 \mathrm{TeV}$ and $n=3$. We can see that the KK graviton signal slightly prefers the region where the two muons are close together while the background receives a large contri-

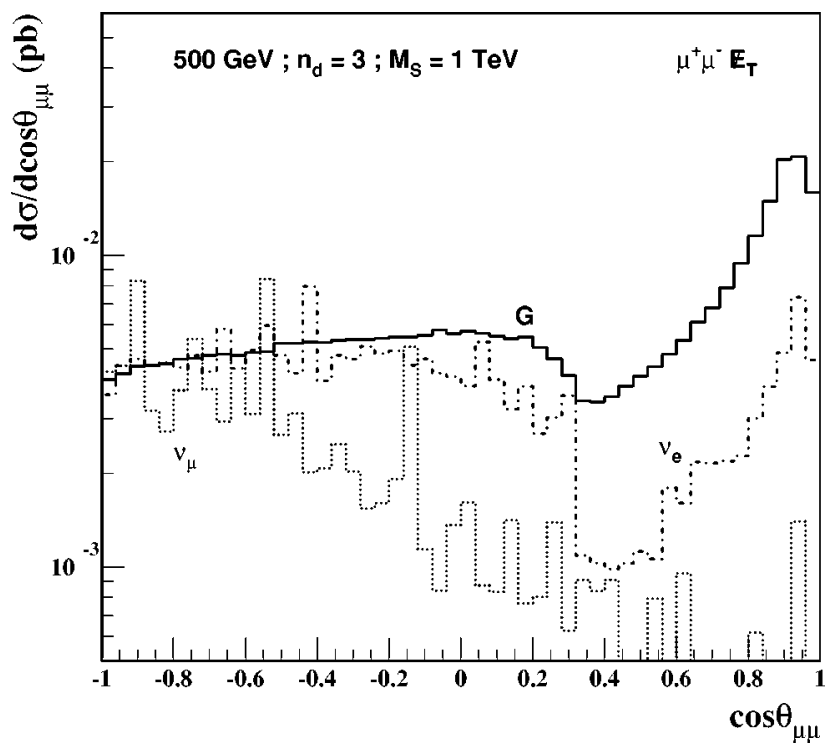

FIG. 3. Distribution of the cosine of the angle between the final state muons originated from the KK graviton radiation and the SM contributions as in Fig. 2 for $\sqrt{s}=500 \mathrm{GeV}$. We assumed $M_{S}$ $=1 \mathrm{TeV}, n=3$, and applied the cuts (C1)-(C5) described in the text. The $\nu_{\tau}$ distribution is not displayed since it is too small. 
TABLE II. Total signal cross sections in fb for the muonic and hadronic channels as a function of the number of extra dimensions, assuming $\sqrt{s}=500 \mathrm{GeV}$ and $M_{S}=1 \mathrm{TeV}$. In the muonic case, we applied the cuts $(\mathrm{C} 1)-(\mathrm{C} 6)$. In the hadronic case, set of cuts $\mathrm{A}$ stands for cuts $(\mathrm{C} 1)-(\mathrm{C} 4)$ and $(\mathrm{C} 7)-(\mathrm{C} 9)$ while set of cuts $\mathrm{B}$ for $(\mathrm{C} 1)-(\mathrm{C} 4)$ and $(\mathrm{C} 10)-(\mathrm{C} 11)$. The SM cross sections are $\sigma_{\mu \mu \mathrm{k}}^{\text {back }}$ $=3.24 \mathrm{fb}$ and $\sigma_{j j E}^{\mathrm{back}}=285$. (8.13) fb for cuts A (B).

\begin{tabular}{|c|c|c|c|c|c|c|}
\hline$n$ & 2 & 3 & 4 & 5 & 6 & 7 \\
\hline$\sigma_{\mu \mu t}^{\text {signal }}(\mathrm{fb})$ & 18.7 & 7.46 & 3.05 & 1.27 & 0.537 & 0.230 \\
\hline$\sigma_{j j E}^{\text {signal }}(\mathrm{fb}):$ cut $\mathrm{A}$ & 387 & 123 & 40.7 & 14.1 & 5.06 & 1.87 \\
\hline$\sigma_{j j E}^{\substack{\text { signal } \\
j \in E}}(\mathrm{fb}):$ cut $\mathrm{B}$ & 30.5 & 12.4 & 5.16 & 2.18 & 0.931 & 0.400 \\
\hline
\end{tabular}

bution from muons in opposite hemispheres. Therefore, we further demanded that (C6) $\cos \theta_{\mu \mu}>0$.

We display in Table II the total cross section for the muonic signal after cuts (C1)-(C6) as a function of the number of large extra dimensions for $M_{S}=1 \mathrm{TeV}$. After these cuts, the SM background is reduced to $\sigma_{\mu \mu}^{\text {back }}=3.24 \mathrm{fb}$, a reduction by a factor of more than 20 while the signal is reduced by a factor of less than 3 . Therefore, these cuts enhance considerably the signal and extend the attainable bounds for the number of extra dimensions up to 6 .

In the hadronic case the main background is $e^{+} e^{-}$ $\rightarrow q \bar{q}^{\prime} e \bar{\nu}$. This background is mainly due to We $\nu$ production with the $W$ further decaying in $q \bar{q}^{\prime}$. Thus a cut in the invariant mass of the jet pair near the $W$ mass can reduce substantially this background. However, the signal cross section peaks at the $Z$ mass, as we can see from Fig. 4. Nevertheless, we found a cut in the jet pair invariant mass that suppresses

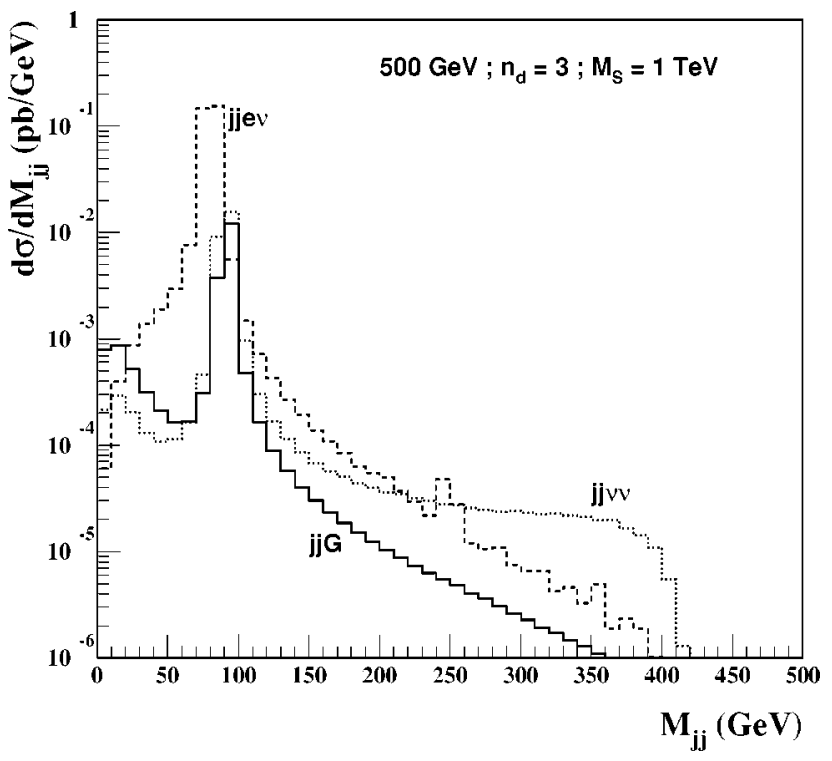

FIG. 4. Dijet invariant mass $\left(M_{j j}\right)$ spectrum originating from the KK graviton radiation (solid line) and the SM contributions to the hadronic case $q \bar{q}^{\prime} e \nu$ (dashed) and $q \bar{q} \nu \nu$ (dotted) for $\sqrt{s}$ $=500 \mathrm{GeV}$. We assumed $M_{S}=1 \mathrm{TeV}, n=3$, and applied the acceptance cuts $(\mathrm{C} 1)-(\mathrm{C} 4)$.

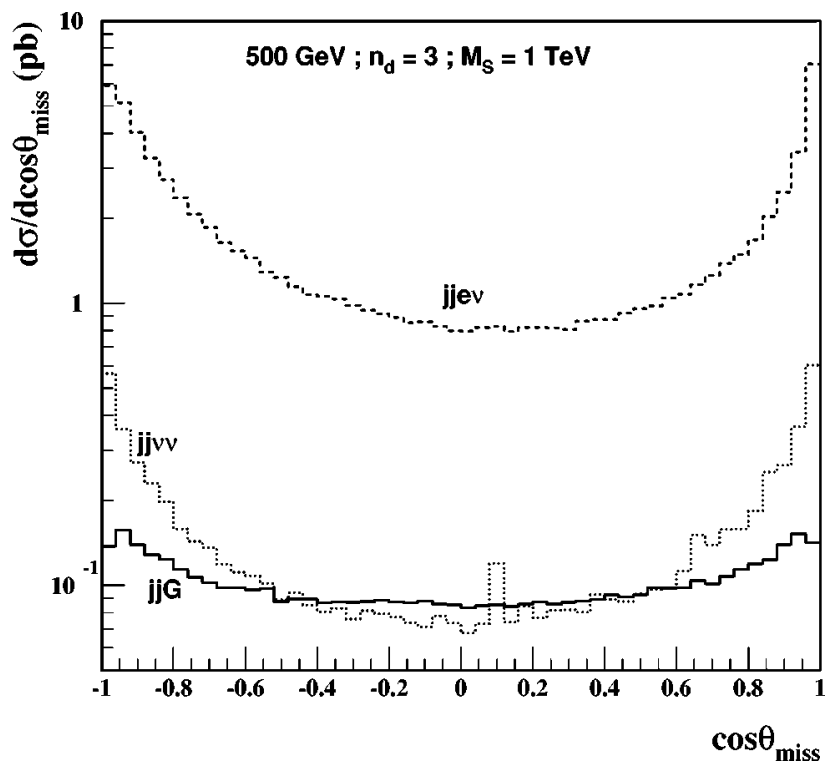

FIG. 5. Missing momentum polar angle $\left(\cos \theta_{\text {miss }}\right)$ spectrum coming from KK graviton radiation and the SM contributions to the hadronic case. We assumed $\sqrt{s}=500 \mathrm{GeV}, M_{S}=1 \mathrm{TeV}, n=3$, and applied the acceptance cuts $(\mathrm{C} 1)-(\mathrm{C} 4)$. The lines follow the conventions as in Fig. 4.

the background while exhibiting a good efficiency for the signal [23]: (C7) $M_{j j}<35 \mathrm{GeV}$ or $M_{j j}>85 \mathrm{GeV}$.

In the background $q \bar{q}^{\prime} e \bar{\nu}$, a large amount of the missing energy is carried by the $e^{ \pm}$escaping through the beam pipe. To illustrate this fact, we present in Fig. 5 the distribution of the cosine of the polar angle of the missing momentum after applying the acceptance cuts $(\mathrm{C} 1)-(\mathrm{C} 4)$, which suggested the introduction of the following cut: (C8) $\left|\cos \theta_{\text {miss }}\right|<0.8$.

After these cuts the $q \bar{q}^{\prime} \nu \bar{\nu}$ total cross section is comparable with $q \bar{q}^{\prime} e \bar{\nu}$; see Fig. 6 which was obtained applying cuts (C1) $-(\mathrm{C} 4)$ and (C7), (C8) for $\sqrt{s}=500 \mathrm{GeV}, M_{S}$ $=1 \mathrm{TeV}$, and $n=3$. The $q \bar{q}^{\prime} \nu \bar{\nu}$ reaction presents a peak in the missing invariant mass $\left(M_{\text {miss }}\right)$ distribution near the $Z$ mass. In order to reduce this background we imposed the conservative cut (C9) $M_{\text {miss }}>200 \mathrm{GeV}$.

The effects of cuts $(\mathrm{C} 1)-(\mathrm{C} 4)$ and $(\mathrm{C} 7)-(\mathrm{C} 9)$ in the signal cross section are shown in Table II, where we can see that the total background is reduced from $\sigma_{j j k}^{\text {back }}=3562$ to $284.7 \mathrm{fb}$, while the signal is reduced by a factor of two at most, resulting in significant enhancement of signal over background. The effects of cuts on the signal are larger for smaller number of dimensions since the cross section for each KK mode goes as $M_{S}^{-n-2} m_{G}^{n-1}$, with $m_{G}$ being the KK mode mass. However, for $n \geqslant 5$ the ratio $S / B$ is lower than 0.1 , which means that we need a precise background estimation. In order to have a better $S / B$ ratio we imposed more stringent cuts. We found out that requiring $(\mathrm{C} 10)$ a harder missing invariant mass of $M_{\text {miss }}>300 \mathrm{GeV}(\mathrm{C} 11)$ and an acoplanarity cut of $\cos \theta_{j j}>0.8$, produces the results shown in the last line of Table II. As we can see, even for $n=6$ we have $S / B>0.1$. 


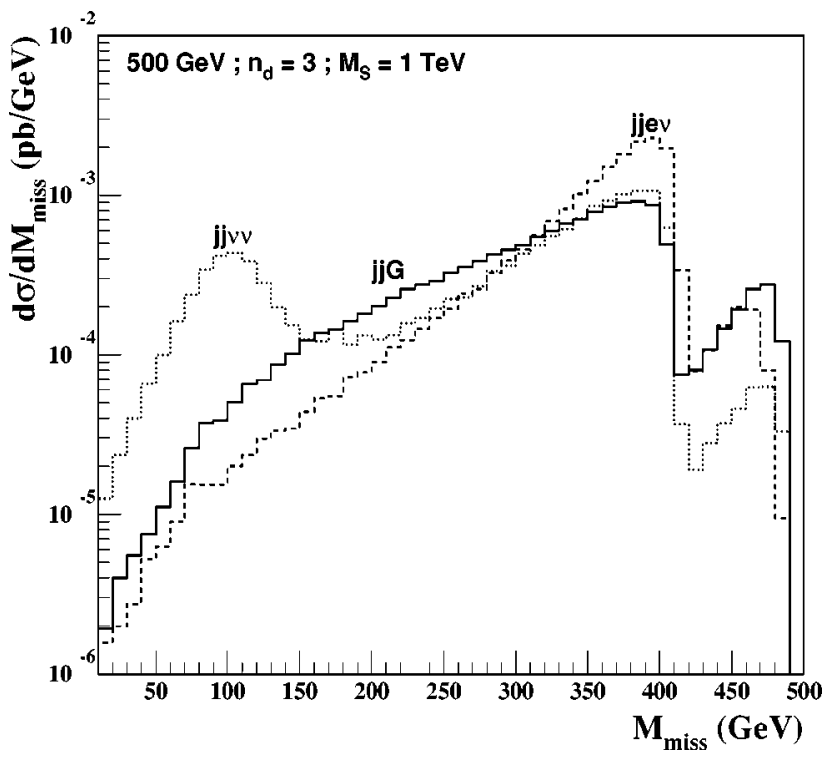

FIG. 6. Missing invariant mass $\left(M_{\text {miss }}\right)$ spectrum originating from KK graviton radiation and the SM contributions to the hadronic case. We assumed $\sqrt{s}=500 \mathrm{GeV}, M_{S}=1 \mathrm{TeV}, n=3$, and applied the acceptance cuts $(\mathrm{C} 1)-(\mathrm{C} 4)$ plus the kinematical cuts (C7),(C8). The lines follow the convention as in Fig. 4.

\section{RESULTS AND DISCUSSIONS}

In this work we studied the potential of $e^{+} e^{-}$colliders to probe the quantum gravity scale $M_{S}$ via the KK graviton emission associated with two fermions (muons and quarks). We considered a LC with a center-of-mass energy $\sqrt{s}$ $=500 \mathrm{GeV}$ and three different integrated luminosities $\mathcal{L}$ $=50,200$, and $500 \mathrm{fb}^{-1}$. We derived the constraints on $M_{S}$ assuming that no deviation from the SM predictions was observed. For a given integrated luminosity $\mathcal{L}$, the statistical significance of the signal is

$$
\frac{\sigma^{\text {signal }}}{\sqrt{\sigma^{\text {back }}}} \sqrt{\mathcal{L}}
$$

where $\sigma^{\text {back }}$ is the total SM background cross section and $\sigma^{\text {signal }}$ the total signal cross section; see Sec. III. Since the quantum gravity signal does not interfere with the SM backgrounds, the KK graviton emission cross section is proportional to $M_{S}^{-n-2}$ and we can write the signal cross section as

$$
\sigma^{\text {signal }}\left(M_{S}, \sqrt{s}\right)=\frac{1}{M_{S}^{n+2}} \sigma^{\text {signal }}(1 \mathrm{TeV}, \sqrt{s}) \text {, }
$$

where $M_{S}$ is given in $\mathrm{TeV}$. Notice that $\sigma^{\text {signal }}$ depends upon the number of extra dimensions $n$ due to the sum over the KK modes. Therefore, one can obtain the 2 (5) $\sigma$ bounds on $M_{S}$ from Eqs. (7) and (8) as

$$
M_{S} \leqslant\left(\frac{\sqrt{\mathcal{L}} \sigma^{\text {signal }}(1 \mathrm{TeV}, \sqrt{s})}{2(5) \sqrt{\sigma^{\text {back }}}}\right)^{1 /(n+2)} .
$$

We present in Table III the $2 \sigma$ and $5 \sigma$ attainable bounds on $M_{S}$ for several choices of $n$, taking into account the muonic, hadronic, and combined channels and using Eq. (9) and the results in Table II. The combined limits were obtained requiring $S / B \geqslant 0.1$, except for $n=7$, and we used the $\chi^{2}$ formalism, i.e., the sum of muonic and hadronic $\chi^{2}$ should result in a $\Delta \chi^{2} \simeq 4$ (25) to be consistent with 2 (5) $\sigma$ limits as prescribed in Ref. [24]. Notice that the hadronic bounds are slightly better than the muonic ones for $n \leqslant 5$ and that for small values of $n$, the set of cuts A gives a better significance even though $S / B$ is bigger for the B cuts.

Our bounds are comparable with the ones derived from the analysis of the associated KK graviton production with a $Z$ in [17], however, our simulations of the signal and backgrounds are more detailed, e.g., we take into account the

TABLE III. 2 (5) $\sigma$ limits in $\mathrm{TeV}$ on the quantum gravity scale $M_{S}$ in $\mathrm{TeV}$ as a function of the number of extra dimensions $n$ for a $500 \mathrm{GeV}$ LC with luminosities $\mathcal{L}=50,200$, and $500 \mathrm{fb}^{-1}$. We present the results

\begin{tabular}{|c|c|c|c|c|c|c|c|}
\hline$n$ & & 2 & 3 & 4 & 5 & 6 & 7 \\
\hline \multirow{3}{*}{ muonic } & $50 \mathrm{fb}^{-1}$ & $2.46(1.96)$ & $1.71(1.42)$ & $1.35(1.16)$ & $1.14(1.00)$ & $1.01(0.90)$ & $0.92(0.83)$ \\
\hline & $200 \mathrm{fb}^{-1}$ & $2.93(2.33)$ & $1.97(1.64)$ & $1.51(1.30)$ & $1.26(1.10)$ & $1.10(0.98)$ & $0.99(0.89)$ \\
\hline & $500 \mathrm{fb}^{-1}$ & $3.28(2.61)$ & $2.15(1.79)$ & $1.63(1.40)$ & $1.34(1.18)$ & $1.16(1.04)$ & $1.04(0.94)$ \\
\hline \multirow{6}{*}{ hadronic } & $50 \mathrm{fb}^{-1}$ & $3.00(2.39)$ & $1.91(1.59)$ & $1.43(1.23)$ & $1.17(1.02)$ & $1.01(0.90)$ & $0.90(0.81)$ \\
\hline & $200 \mathrm{fb}^{-1}$ & $3.57(2.84)$ & $2.20(1.83)$ & $1.60(1.38)$ & $1.29(1.13)$ & $1.10(0.98)$ & $0.97(0.88)$ \\
\hline & $500 \mathrm{fb}^{-1}$ & $4.00(3.18)$ & $2.41(2.01)$ & $1.73(1.49)$ & $1.38(1.21)$ & $1.16(1.04)$ & $1.02(0.92)$ \\
\hline & $50 \mathrm{fb}^{-1}$ & $2.48(1.97)$ & $1.73(1.44)$ & $1.36(1.17)$ & $1.15(1.01)$ & $1.02(0.91)$ & $0.93(0.84)$ \\
\hline & $200 \mathrm{fb}^{-1}$ & $2.95(2.35)$ & $1.99(1.65)$ & $1.53(1.31)$ & $1.27(1.12)$ & $1.11(0.99)$ & $1.00(0.90)$ \\
\hline & $500 \mathrm{fb}^{-1}$ & $3.31(2.63)$ & $2.18(1.81)$ & $1.65(1.42)$ & $1.36(1.19)$ & $1.18(1.05)$ & $1.05(0.95)$ \\
\hline \multirow{3}{*}{ combined } & $50 \mathrm{fb}^{-1}$ & $3.07(2.44)$ & $1.97(1.64)$ & $1.48(1.27)$ & $1.20(1.06)$ & $1.06(0.94)$ & $0.96(0.86)$ \\
\hline & $200 \mathrm{fb}^{-1}$ & $3.65(2.91)$ & $2.26(1.88)$ & $1.66(1.42)$ & $1.33(1.17)$ & $1.15(1.03)$ & $1.03(0.93)$ \\
\hline & $500 \mathrm{fb}^{-1}$ & $4.10(3.26)$ & $2.48(2.06)$ & $1.79(1.54)$ & $1.42(1.25)$ & $1.22(1.09)$ & $1.09(0.98)$ \\
\hline
\end{tabular}
for the muonic, hadronic with the two cut selections as in Table II, and combined channels. For the latter we required $S / B \geqslant 0.1$, except for $n=7$. 
$\gamma^{*} / Z^{*}$ interference, more realistic cuts, and reducible backgrounds. Moreover, we were able to extend the bounds to all values of $n$ in contrast with the results in Ref. [17] which are valid only for $n=2$. It is important to notice that for $n=7$ the ratio $S / B \simeq 0.05$ which means that the bounds presented here should be taken with a grain of salt since we need a more careful study of the backgrounds in order to take seriously the $n=7$ limits into account.

We can also see from Table III that a $500 \mathrm{GeV}$ LC will be able to probe the quantum gravity scale $M_{S}$ above the new gravitational direct experimental limit $M_{S} \geqslant 3.5 \mathrm{TeV}$ for $n$ $=2$ [3] provided its integrated luminosity is larger than $200 \mathrm{fb}^{-1}$. Present collider limits are, in general, less stringent than this one. For instance, graviton direct production at the CERN $200 \mathrm{GeV} e^{+} e^{-}$collider LEP gives 95\% C.L. limits of $M_{S} \geqslant 1.02-1.25 \mathrm{TeV}$ for $n=2$ [25], and searches for virtual graviton effects lead to $95 \%$ C.L. limits of $M_{S}$ $\geqslant 0.75-1.3 \mathrm{TeV}$ for any number of extra dimensions depending on the LEP experiment [25]. The Fermilab Tevatron DØ experiment presented a $95 \%$ C.L. bound of $M_{S}$ $\geqslant 1.37 \mathrm{TeV}$ for $n=2$ based on searches for virtual graviton effects on dielectron or diphoton systems [26].

We should compare our limits to the ones obtained from alternative signatures at a $500 \mathrm{GeV} e^{+} e^{-}$linear collider. The most significant bounds come from $\gamma G$ production giving a $5 \sigma$ limit of $M_{S} \geqslant 3.66 \mathrm{TeV}$ [27], and gauge boson pair production $(V V)$ giving a $2 \sigma$ limit of $M_{S}$ $\geqslant 2.8(3.0)(3.2) \mathrm{TeV}$ for $V=W(Z)(\gamma)[14]$. These results are comparable to the ones presented in Table III, however, we expect that our signature can give direct information about the graviton spin through the study of the angular distributions of the final particles.

In brief, we showed in detail that $500 \mathrm{GeV}$ LC with an integrated luminosity of $500 \mathrm{fb}^{-1}$ will be able to exclude $M_{S}$ up to 4.1 (1.1) $\mathrm{TeV}$ for $n=2$ (7). Although our results are well above the actual experimental limits from LEP and Tevatron, they are a factor of 2 less stringent than the expected ones from the CERN Large Hadron Collider (LHC) [15], however, the LC leads to cleaner and easier to reconstruct events. Moreover, at the LHC there is an ambiguity on how to unitarize the cross sections since at very high partonparton center-of-mass energies the subprocesses involving KK gravitons violate unitarity. The signals studied here are free from this ambiguity as we have the direct production of the graviton. In addition it might be possible to probe the spin of the graviton looking at angular distributions [28].

\section{ACKNOWLEDGMENTS}

We would like to thank D. Zeppenfeld for discussions. This research was supported in part by Conselho Nacional de Desenvolvimento Científico e Tecnológico (CNPq), by Fundação de Amparo à Pesquisa do Estado de São Paulo (FAPESP), and by Programa de Apoio a Núcleos de Excelência (PRONEX).
[1] N. Arkani-Hamed, S. Dimopoulos, and G. Dvali, Phys. Lett. B 429, 263 (1998); I. Antoniadis, N. Arkani-Hamed, S. Dimopoulos, and G. Dvali, ibid. 436, 257 (1998); I. Antoniadis and C. Bachas, ibid. 450, 83 (1999).

[2] N. Arkani-Hamed, S. Dimopoulos, and G. Dvali, Phys. Rev. D 59, 086004 (1999).

[3] C.D. Hoyle et al., Phys. Rev. Lett. 86, 1418 (2001).

[4] See, e.g., Modern Kaluza-Klein Theories, edited by T. Appelquist, A. Chodos, and P. Freund (Addison-Wesley, New York, 1987).

[5] E. Witten, Nucl. Phys. B443, 85 (1995); P. Hořava and E. Witten, ibid. B460, 506 (1996); B475, 94 (1996).

[6] For recent discussions, see, for example, TASI96: Fields, Strings and Duality, edited by C. Efthimiou and B. Greene (World Scientific, Singapore, 1997), and references therein.

[7] I. Antoniadis, Phys. Lett. B 246, 377 (1990); J. Lykken, Phys. Rev. D 54, 3693 (1996); K. Dienes, E. Dudas, and T. Ghergetta, Phys. Lett. B 436, 55 (1998); G. Shiu and S.-H.H. Tye, Phys. Rev. D 58, 106007 (1998); Z. Kakushadze and S.-H.H. Tye, Nucl. Phys. B548, 180 (1999); L.E. Ibáñez, C. Muñoz, and S. Rigolin, ibid. B553, 43 (1999).

[8] L.N. Chang, O. Lebedev, W. Loinaz, and T. Takeuchi, Phys. Rev. Lett. 85, 3765 (2000).

[9] J. Hewett, Phys. Rev. Lett. 82, 4765 (1999).

[10] G.F. Giudice, R. Rattazzi, and J.D. Wells, Nucl. Phys. B544, 3 (1999); E.A. Mirabelli, M. Perelstein, and M.E. Peskin, Phys. Rev. Lett. 82, 2236 (1999).
[11] P. Mathews, S. Raychaudhuri, and K. Sridhar, Phys. Lett. B 450, 343 (1999); T. Rizzo, hep-ph/9902273.

[12] T. Han, J. Lykken, and R.-J. Zhang, Phys. Rev. D 59, 105006 (1999).

[13] P. Mathews, S. Raychaudhuri, and K. Sridhar, Phys. Lett. B 455, 115 (1999); J. High Energy Phys. 07, 008 (2000); T. Rizzo, Phys. Rev. D 59, 115010 (1999); 60, 075001 (1999); 60, 115010 (1999); S.Y. Choi et al., ibid. 60, 013007 (1999); C. Balazs, D.A. Dicus, H.-J. He, W.W. Repko, and C.-P. Yuan, Phys. Rev. Lett. 83, 2112 (1999); G. Shiu, R. Shrock, and S.-H.H. Tye, Phys. Lett. B 458, 274 (1999); K.Y. Lee, H.S. Song, and J. Song, ibid. 464, 82 (1999); Hooman Davoudiasl, Phys. Rev. D 60, 084022 (1999); K. Cheung, Phys. Lett. B 460, 383 (1999); Xiao-Gang He, Phys. Rev. D 61, 036007 (2000).

[14] K. Agashe and N.G. Deshpande, Phys. Lett. B 456, 60 (1999).

[15] P. Mathews, S. Raychaudhuri, and K. Sridhar, Phys. Lett. B 450, 343 (1999); O.J.P. Éboli, T. Han, M.B. Magro, and P.G. Mercadante, Phys. Rev. D 61, 094007 (2000); K. Cheung, ibid. 61, 015005 (2000).

[16] T. Han, D. Rainwater, and D. Zeppenfeld, Phys. Lett. B 463, 93 (1999).

[17] K. Cheung and Wai-yee Keung, Phys. Rev. D 60, 112003 (1999).

[18] J.A.M. Vermaseren, math-ph/0010025.

[19] T. Stelzer and W.F. Long, Comput. Phys. Commun. 81, 357 (1994). 
[20] K. Hagiwara, H. Iwasaki, A. Miyamoto, H. Murayama, and D. Zeppenfeld, Nucl. Phys. B365, 544 (1991).

[21] U. Baur, J.A.M. Vermaseren, and D. Zeppenfeld, Nucl. Phys. B375, 3 (1992); A. Aeppli, F. Cuypers, and G.J. van Oldenborgh, Phys. Lett. B 314, 413 (1997).

[22] E.A. Kuraev and V.S. Fadin, Sov. J. Nucl. Phys. 41, 466 (1985).

[23] V. Barger et al., Phys. Rev. D 52, 3815 (1995); O.J.P. Éboli, M.C. Gonzalez-García, and J.K. Mizukoshi, ibid. 58, 034008 (1998).
[24] Particle Data Group, D.E. Groom et al., Eur. Phys. J. C 15, 1 (2000).

[25] G. Landsberg, in "Proceedings of 30th International Conference on High-Energy Physics" (ICHEP 2000), Osaka, Japan, 2000, hep-ex/0009038 and references therein.

[26] D0 Collaboration, B. Abbott et al., Phys. Rev. Lett. 86, 1156 (2001).

[27] M. Besançon, hep-ph/9909364.

[28] O.J.P. Éboli et al. (in preparation). 